\title{
Comparison of different imputation methods from low- to high-density panels using Chinese Holstein cattle
}

\author{
Z. Weng ${ }^{1}$, Z. Zhang ${ }^{1,2}$, Q. Zhang ${ }^{1}$, W. Fu ${ }^{1}$, S. He ${ }^{1}$ and X. Ding ${ }^{1+}$ \\ ${ }^{1}$ Key Laboratory of Animal Genetics, Breeding and Reproduction, Ministry of Agriculture of China, National Engineering Laboratory for Animal Breeding, College of \\ Animal Science and Technology, China Agricultural University, Beijing 100193, China; ${ }^{2}$ Guangdong Provincial Key Lab of Agro-Animal Genomics and Molecular \\ Breeding, College of Animal Science, South China Agricultural University, Guangzhou 510642, China
}

(Received 6 March 2012; Accepted 12 September 2012; First published online 11 December 2012)

Imputation of high-density genotypes from low- or medium-density platforms is a promising way to enhance the efficiency of whole-genome selection programs at low cost. In this study, we compared the efficiency of three widely used imputation algorithms (fastPHASE, BEAGLE and findhap) using Chinese Holstein cattle with Illumina BovineSNP50 genotypes. A total of 2108 cattle were randomly divided into a reference population and a test population to evaluate the influence of the reference population size. Three bovine chromosomes, BTA1, 16 and 28, were used to represent large, medium and small chromosome size, respectively. We simulated different scenarios by randomly masking 20\%,40\%, 80\% and 95\% single-nucleotide polymorphisms (SNPS) on each chromosome in the test population to mimic different SNP density panels. Illumina Bovine3K and Illumina BovineLD (6909 SNPS) information was also used. We found that the three methods showed comparable accuracy when the proportion of masked SNPs was low. However, the difference became larger when more SNPs were masked. BEAGLE performed the best and was most robust with imputation accuracies $>90 \%$ in almost all situations. fastPHASE was affected by the proportion of masked SNPs, especially when the masked SNP rate was high. findhap ran the fastest, whereas its accuracies were lower than those of BEAGLE but higher than those of fastPHASE. In addition, enlarging the reference population improved the imputation accuracy for BEAGLE and findhap, but did not affect fastPHASE. Considering imputation accuracy and computational requirements, BEAGLE has been found to be more reliable for imputing genotypes from low- to high-density genotyping platforms.

Keywords: missing genotypes, SNPs, imputation

\section{Implication}

In this study, we compared the efficiency of three widely used imputation algorithms, fastPHASE, BEAGLE and findhap, using Chinese Holstein cattle genotyped with the Illumina BovineSNP50 BeadChip (Illumina Inc., San Diego, CA, USA). We found that the three methods showed comparable accuracy when the proportion of masked single-nucleotide polymorphisms (SNPs) was low. However, the difference became larger when more SNPs were masked. BEAGLE performed the best and was most robust with imputation accuracies $>90 \%$ in almost all situations. Our research is helpful for imputing genotypes from low- to high-density genotyping platforms.

\footnotetext{
† E-mail: xding@cau.edu.cn
}

\section{Introduction}

The recent development of high-throughput systems for genotyping single-nucleotide polymorphisms (SNPs) has led to an extraordinary amount of research activities. With the availability of low- (Illumina Bovine $3 \mathrm{~K}$ or BovineLD, San Diego, CA, USA), medium- (Illumina BovineSNP50, San Diego, CA, USA) and high-density (Illumina BovineHD, San Diego, CA, USA) SNP platforms in dairy cattle, imputation of high-density genotypes from low- or medium-density platforms is a promising way to promote genomic selection based on different SNP platforms in dairy cattle (Weigel et al., 2010; Zhang and Druet, 2010).

Several imputation methods based on various statistical models, such as the haplotype clustering algorithm (Scheet and Stephens, 2006), the hidden Markov model (HMM; Browning 
and Browning, 2007), the Expectation Maximization (EM) algorithm (Qin et al., 2002; Scheet and Stephens, 2006) and the Markov Chain model (Li et al., 2010), have been proposed. The accuracy of imputing missing genotypes using different haplotype reconstruction methods has been mostly compared using real data in humans (Marchini et al., 2007; Pei et al., 2008; Nothnagel et al., 2009; Shriner et al., 2010; Weigel et al., 2010). In dairy cattle, studies have been conducted to investigate the accuracy of imputation from low- to high-density using real SNP data sets from cattle populations (Weigel et al., 2010; Zhang and Druet, 2010; Berry and Kearney, 2011); however, comparisons between prevailing methods commonly used in human populations have not been carried out in these studies.

The objective of this study was to investigate the imputation accuracy and computational requirements of three widely used imputation algorithms in animals, fastPHASE (Scheet and Stephens, 2006), BEAGLE (Browning and Browning, 2007) and findhap (VanRaden et al., 2011). We simulated scenarios with different masking SNP rates using Illumina BovineSNP50 genotypes of Chinese Holstein cattle to mimic different density SNP panels in order to evaluate their performance of imputing genotypes from low to high density. Factors affecting imputation accuracy were also studied. The results of this study are expected to be helpful in guiding the application of imputation methods in genomic selection and genome-wide association studies in Chinese dairy cattle, especially when using low- or medium-density platforms to select parents of the subsequent generation.

\section{Material and methods}

\section{Data}

A total of 2180 Chinese Holstein cattle (87 bulls and 2093 cows) were genotyped using the Illumina BovineSNP50 BeadChip (Illumina Inc.). The SNP data were edited using the following procedures: (1) SNP genotypes with Mendelian inheritance errors were treated as missing; (2) SNPs with call rates $<0.90$ or minor allele frequency (MAF) $<0.01$ were removed; and (3) cattle with call rates $<0.90$ were deleted. After selection, 2108 cattle (87 bulls and 2021 cows) and 45727 SNPs remained on all chromosomes; the proportion of missing SNPs was low, $0.58 \%$ on average after quality control. Pedigree errors of 241 sire-daughter pairs were detected on the basis of the 45727 remaining SNPs using program Cervus (Marshall et al., 1998). After pedigree correction, 13 of the 87 bulls were sires of all 2093 genotyped cows (each bull had 83 to 358 daughters with an average of 150), and the other 74 bulls did not have any genotyped progeny; there were no sibship or parent/grandparent relationships among 87 bulls.

The 2108 cattle were divided into a reference population and a test population to evaluate the performance of different imputation methods. The reference population consisted of all bulls and half of the cows (randomly selected), whereas the test population consisted of the remaining half of the cows. The individuals in the reference population kept their original genotype data, whereas in the test population various proportions of SNP genotypes were masked. The masked SNPs were then imputed from the genotype information provided by the reference population. Three chromosomes, BTA1, 16 and 28, were used to represent large, medium and small chromosome size, respectively.

To make a comprehensive comparison of different imputation methods, different proportions $(20 \%, 40 \%, 80 \%$ and $95 \%$ ) of masked SNPs in the test population were simulated by randomly masking the existing genotypes on the three chromosomes. For the masking proportion of $95 \%$ on BTA16, three additional structures/sizes of the reference population were considered: (1) 87 bulls only; (2) 87 bulls and 101 cows; and (3) 87 bulls and 401 cows. The corresponding remaining cows for each reference population would be the test population. For each scenario, 10 replicates were simulated, and, in each replication, SNPs were randomly masked at the assigned proportion.

At the moment, Illumina has marketed two low-density Bovine SNP chips. The original 3k (Bovine3K) has been replaced by the BovineLD with 6909 (7k) SNPs (Boichard et al., 2012). We used the real SNP information on $3 \mathrm{k}$ and $7 \mathrm{k}$ chips, in which $\sim 5 \%$ and $13 \%$ SNPs on a 50k chip (BovineSNP50) are drawn equally from each chromosome, respectively. Therefore, the results from BTA1, 16 and 28 are still representative for the comparison of different imputation methods.

\section{Imputation methods}

Three widely used imputation methods were evaluated in our study:

(1) fastPHASE: fastPHASE uses a localized haplotype clustering algorithm (Scheet and Stephens, 2006). It assumes that haplotypes of individuals in the population tend to cluster into groups of closely related or similar haplotypes within a short region of a chromosome. This method allows memberships of clusters to vary along the chromosome based on an HMM. Missing genotypes are sampled on the basis of allele frequencies estimated from reference haplotypes, and then an EM algorithm is used to estimate parameter values to infer missing genotypes. The computing time of the fastPHASE algorithm increases linearly with the number of ungenotyped individuals and the number of haplotype clusters (Weigel et al., 2010). The clusters represent groups of closely related haplotypes. Its value is defined by parameter $\mathrm{K}$ in fastPHASE v1.4. In our study, two values of $K, 20$ and 30, were applied.

(2) BEAGLE: BEAGLE is also a localized haplotype clustering-based algorithm (Browning and Browning, 2007). First, it gathers haplotype clusters at each marker and defines an HMM to find the most likely haplotype pairs based on the known genotypes of each individual. At that time, the most likely genotype at the missing genotype loci could be deduced from final haplotype pairs. Both BEAGLE and fastPHASE use an HMM approach to cluster haplotypes. However, some slight differences exist between them. First, fastPHASE estimates parameters for cluster configuration using an EM algorithm, whereas BEAGLE uses empirical frequencies. Second, unlike fastPHASE, which relies on a fixed number of haplotype clusters to form underlying 
hidden states in the Markov Chain, BEAGLE allows the cluster number to dynamically change to better fit localized linkage disequilibrium (LD) patterns (Pei et al., 2008). According to the recommendation by Browning and Browning (2007), the default 10 iterations are enough to obtain a high accuracy for BEAGLE. In this study, the program BEAGLE 3.3 was used and the number of iterations of the phasing algorithm was set to 20 .

(3) findhap: Different from fastPHASE and BEAGLE, which mainly deal with a sample of unrelated individuals, a pedigree-based algorithm is implemented in findhap v2.0 (VanRaden et al., 2011). The program is designed to integrate the population with pedigree haplotyping. The process starts with dividing each chromosome into segments and creating a list of haplotypes from genotypes in the first round. In continued iterations, earlier created genotypes are matched again using haplotypes that occurred later. The first two iterations mainly focus on population haplotyping: only the highest density genotypes are used in the first iteration, and then all genotypes are used in the second iteration. After population haplotyping, haplotypes are matched by using both pedigree and population in the following two iterations. In this study, the program was run with 20 iterations to capture a higher accuracy. As reported by VanRaden et al. (2011), the length of haplotype segments could affect the imputation accuracy and computing time. Here the maximum and minimum lengths of haplotype segments were defined as 100 and 50 for the scenarios with $20 \%, 40 \%$ and $80 \%$ missing SNPs and as 600 and 75 for the scenario with $95 \%$ missing SNPs, respectively.

\section{Accuracy of imputation}

In each replicate, imputation accuracy was measured as the percentage of correctly imputed genotypes in the test population. A correctly imputed genotype was defined as complete concordance between the original and the imputed genotype of a masked SNP locus. We calculated average imputation accuracy rate and standard error across all masked loci on each chromosome for 10 replicates.

Computing time of the three programs was measured in hours for fastPHASE and BEAGLE and in seconds for findhap on a Linux cluster (2.0 GHZ Intel Xeon processor and Redhat Enterprise 4.1) with three computation nodes.

\section{Results}

The detailed information of SNPs on BTA1, BTA16 and BTA28 is listed in Table 1. Generally, the three chromosomes have

Table 1 Genomic information of three bovine chromosomes in Chinese Holstein

\begin{tabular}{lcrccc}
\hline \hline Chromosome & No. SNP & $\begin{array}{c}\text { Length } \\
(\mathrm{Mb})\end{array}$ & $\begin{array}{c}\text { Call } \\
\text { rate }\end{array}$ & $\begin{array}{c}\text { Average } \\
\text { interval }(\mathrm{kb})\end{array}$ & LD $\left(r^{2}\right)$ \\
\hline 1 & 2877 & 161.06 & 0.98 & 55.98 & 0.27 \\
16 & 1367 & 77.82 & 0.98 & 56.92 & 0.28 \\
28 & 823 & 46.00 & 0.98 & 55.89 & 0.21 \\
\hline \hline
\end{tabular}

SNP = single-nucleotide polymorphism; $L D=$ linkage disequilibrium. almost the same average interval between SNPs (about $56 \mathrm{~kb}$ apart), equal average SNP call rate (0.98) and similar average LD level $\left(r^{2}\right)$ between adjacent SNPs (0.21 to 0.28$)$. After randomly masking $20 \%$ to $95 \%$ of SNPs on each chromosome in the test population, the number of available SNPs ranged from 2301 to 143 for BTA1, from 1093 to 68 for BTA16 and from 658 to 41 for BTA28.

\section{Comparison of imputation accuracy}

The results of comparison of BEAGLE, fastPHASE and findhap are shown in Table 2. In general, for all three methods, the imputation accuracy decreased along with the increase in the proportion of missing SNPs. BEAGLE performed best and yielded accuracies $>90 \%$ in all cases. When the proportion of masked SNPs was low, for example, $20 \%$ or $40 \%$, fastPHASE performed slightly better compared with findhap. However, when more SNPs were masked, fastPHASE performed worse than findhap. In particular, when the proportion of masked SNPs increases to $95 \%$, the accuracy from fastPHASE is $20 \%$ and $14 \%$ lower than that of BEAGLE and findhap on average, respectively.

In fastPHASE, haplotype clusters represent groups of closely related haplotypes. In this study, we set 20 and 30 haplotype clusters to determine their influence on the imputation accuracy of fastPHASE. As shown in Table 2, the imputation accuracy of fastPHASE could be improved by increasing the number of haplotype clusters, although the improvement was very slight in all situations; in addition, the computing time was doubled as shown in Table 3.

For each scenario, we made 10 replicates, and it is demonstrated that all imputation methods yield very small standard error in most situations, showing that these methods perform robustly in genotype imputation. Moreover, for each method, the imputation accuracies for the three chromosomes were almost identical in the case with the same proportion of masked SNPs. For instance, the accuracies for BTA1, BTA16 and BTA28 from BEAGLE were $0.986,0.984$ and 0.981 , respectively, when $20 \%$ SNPs were masked. Similarly, fastPHASE and findhap showed similar robustness as well. This is expected as the average SNP interval, SNP call rate and LD level on the three chromosomes are almost equal (see Table 1).

Table 4 shows the performance of the three methods in imputing genotypes from Illumina Bovine3K (3k) and BovineLD (7k) to BovineSNP50 (50k). For all three approaches, accuracy of imputing genotypes from $3 \mathrm{k}$ to $50 \mathrm{k}$ was higher than for the scenario of randomly masking 95\% SNPs. This could be because high MAF and higher concentrations at the ends of the chromosomes were criteria for SNP selection for the Illumina chips. When imputing genotypes from $7 \mathrm{k}$ to $50 \mathrm{k}$, the accuracy of BEAGLE was $>0.96$ across three chromosomes, more accurate than that from $3 \mathrm{k}$ to $50 \mathrm{k}$. This is similar to reports from Boichard et al. (2012). They reported that the accuracy of imputing genotypes from $7 \mathrm{k}$ to 50k was about 0.97 for Australian Holstein without a sire in the reference population (Boichard et al., 2012). The performance of fastPHASE and findhap showed a similarity 
Weng, Zhang, Zhang, Fu, He and Ding

Table 2 Average imputation accuracy from BEAGLE, fastPHASE and findhap at different masked SNPs rate based on 10 replicates

\begin{tabular}{|c|c|c|c|c|c|}
\hline Chromosome & $\begin{array}{l}\text { Proportion of masked SNP } \\
\text { genotypes in the test population }\end{array}$ & $\begin{array}{l}\text { BEAGLE } 3.3 \\
\text { (20 iterations) }\end{array}$ & $\begin{array}{c}\text { fastPHASE } 1.4 \\
\text { (20 haplotype clusters) }\end{array}$ & $\begin{array}{c}\text { fastPHASE } 1.4 \\
\text { (30 haplotype clusters) }\end{array}$ & $\begin{array}{l}\text { findhap } 2.0 \\
\text { (20 iterations) }\end{array}$ \\
\hline \multirow[t]{4}{*}{ BTA1 } & 0.20 & $0.986 \pm 0.001$ & $0.970 \pm 0.001$ & $0.981 \pm 0.001$ & $0.951 \pm 0.001$ \\
\hline & 0.40 & $0.984 \pm 0.001$ & $0.967 \pm 0.002$ & $0.979 \pm 0.001$ & $0.955 \pm 0.001$ \\
\hline & 0.80 & $0.972 \pm 0.001$ & $0.913 \pm 0.003$ & $0.931 \pm 0.003$ & $0.952 \pm 0.002$ \\
\hline & 0.95 & $0.918 \pm 0.004$ & $0.724 \pm 0.005$ & $0.721 \pm 0.004$ & $0.869 \pm 0.004$ \\
\hline \multirow[t]{4}{*}{ BTA16 } & 0.20 & $0.984 \pm 0.001$ & $0.967 \pm 0.002$ & $0.979 \pm 0.002$ & $0.942 \pm 0.003$ \\
\hline & 0.40 & $0.983 \pm 0.001$ & $0.963 \pm 0.001$ & $0.975 \pm 0.002$ & $0.949 \pm 0.002$ \\
\hline & 0.80 & $0.968 \pm 0.001$ & $0.898 \pm 0.005$ & $0.919 \pm 0.005$ & $0.940 \pm 0.006$ \\
\hline & 0.95 & $0.901 \pm 0.007$ & $0.709 \pm 0.008$ & $0.715 \pm 0.007$ & $0.842 \pm 0.009$ \\
\hline \multirow[t]{4}{*}{ BTA28 } & 0.20 & $0.981 \pm 0.001$ & $0.957 \pm 0.002$ & $0.971 \pm 0.002$ & $0.943 \pm 0.003$ \\
\hline & 0.40 & $0.978 \pm 0.001$ & $0.952 \pm 0.002$ & $0.966 \pm 0.003$ & $0.950 \pm 0.003$ \\
\hline & 0.80 & $0.960 \pm 0.001$ & $0.889 \pm 0.005$ & $0.915 \pm 0.005$ & $0.933 \pm 0.008$ \\
\hline & 0.95 & $0.913 \pm 0.009$ & $0.699 \pm 0.006$ & $0.700 \pm 0.010$ & $0.889 \pm 0.029$ \\
\hline
\end{tabular}

SNP = single-nucleotide polymorphism.

Table 3 Computing time of BEAGLE, fastPHASE and findhap at different masked SNPs rate based on 10 replicates

\begin{tabular}{|c|c|c|c|c|c|}
\hline Chromosome & $\begin{array}{l}\text { Proportion of masked SNP } \\
\text { genotypes in test population }\end{array}$ & $\begin{array}{l}\text { BEAGLE } 3.3(20 \\
\text { iterations) (h) }\end{array}$ & $\begin{array}{l}\text { fastPHASE } 1.4 \text { ( } 20 \\
\text { haplotype clusters) (h) }\end{array}$ & $\begin{array}{c}\text { fastPHASE } 1.4 \text { (30 } \\
\text { haplotype clusters) (h) }\end{array}$ & $\begin{array}{l}\text { findhap } 2.0 \text { (20 } \\
\text { iterations) (s) }\end{array}$ \\
\hline \multirow[t]{4}{*}{ BTA1 } & 0.20 & $0.893 \pm 0.049$ & $55.892 \pm 0.364$ & $115.196 \pm 0.808$ & $23.333 \pm 2.739$ \\
\hline & 0.40 & $1.244 \pm 0.055$ & $39.123 \pm 0.620$ & $81.920 \pm 0.745$ & $23.111 \pm 2.088$ \\
\hline & 0.80 & $4.681 \pm 0.242$ & $37.140 \pm 0.204$ & $78.893 \pm 0.550$ & $23.222 \pm 1.787$ \\
\hline & 0.95 & $18.531 \pm 0.635$ & $37.583 \pm 0.306$ & $79.090 \pm 0.538$ & $73.889 \pm 3.586$ \\
\hline \multirow[t]{4}{*}{ BTA16 } & 0.20 & $0.434 \pm 0.012$ & $26.327 \pm 0.721$ & $54.788 \pm 0.472$ & $34.000 \pm 0.707$ \\
\hline & 0.40 & $0.662 \pm 0.012$ & $19.832 \pm 0.267$ & $41.899 \pm 0.752$ & $34.444 \pm 0.527$ \\
\hline & 0.80 & $2.990 \pm 0.067$ & $18.001 \pm 0.818$ & $37.449 \pm 0.356$ & $34.333 \pm 0.866$ \\
\hline & 0.95 & $10.024 \pm 0.489$ & $17.908 \pm 0.176$ & $37.738 \pm 0.281$ & $45.778 \pm 1.716$ \\
\hline \multirow[t]{4}{*}{ BTA28 } & 0.20 & $0.207 \pm 0.013$ & $15.686 \pm 0.148$ & $33.420 \pm 0.173$ & $25.889 \pm 2.369$ \\
\hline & 0.40 & $0.333 \pm 0.013$ & $12.702 \pm 0.300$ & $26.570 \pm 0.312$ & $25.444 \pm 1.130$ \\
\hline & 0.80 & $1.632 \pm 0.125$ & $10.474 \pm 0.035$ & $22.640 \pm 0.109$ & $25.444 \pm 0.726$ \\
\hline & 0.95 & $5.830 \pm 0.303$ & $10.939 \pm 0.224$ & $22.550 \pm 0.097$ & $33.778 \pm 1.093$ \\
\hline
\end{tabular}

SNP = single-nucleotide polymorphism.

Table 4 Imputation accuracy from BEAGLE, fastPHASE and findhap at Illumina 3k (Bovine3K) and 7k (BovineLD) chip

\begin{tabular}{llccr}
\hline \hline SNP chip & Chromosome & BEAGLE 3.3 (20 iterations) & fastPHASE 1.4 (20 haplotype clusters) & findhap 2.0 (20 iterations) \\
\hline $3 k$ & BTA1 & 0.943 & 0.775 & 0.910 \\
& BTA16 & 0.930 & 0.745 & 0.883 \\
$7 \mathrm{k}$ & BTA28 & 0.914 & 0.731 & 0.876 \\
& BTA1 & 0.968 & 0.906 & 0.946 \\
& BTA16 & 0.967 & 0.891 & 0.938 \\
& BTA28 & 0.961 & 0.879 & 0.932 \\
\hline \hline
\end{tabular}

SNP = single-nucleotide polymorphism.

tendency, although their accuracies were still lower than that of BEAGLE. In all situations, BEAGLE yielded high and stable accuracy.

\section{Computing time}

All three programs were run on a Linux cluster (CPU 2.0 MHZ) with three computation nodes. BEAGLE and fastPHASE can use only one processor, whereas findhap can utilize a maximum of seven processors simultaneously by implementing a parallel computing algorithm. The mean computing time and standard errors of different imputation algorithms for BTA1, 16 and 28 are listed in Table 3. fastPHASE required much more computing time than the other two methods, and its computing time increased markedly when the number of haplotype clusters increased from 20 to 30 . findhap was the fastest and could accomplish imputation within $1 \mathrm{~min}$ in all cases.

The number of SNP markers also probably influences computing time. For example, BTA1 had 2054 more SNPs 
Table 5 Average imputation accuracy of BEAGLE, fastPHASE and findhap with different reference population size in scenario of randomly masking 95\% genotypes on chromosome 16

\begin{tabular}{lcccc}
\hline \hline $\begin{array}{l}\text { Composition of the reference } \\
\text { population }\end{array}$ & $\begin{array}{c}\text { Composition of the test } \\
\text { population }\end{array}$ & $\begin{array}{c}\text { BEAGLE } 3.3(20 \\
\text { iterations) }\end{array}$ & $\begin{array}{c}\text { fastPHASE } 1.4 \text { (20 haplotype } \\
\text { clusters) }\end{array}$ & $\begin{array}{c}\text { findhap 2.0 (20 } \\
\text { iterations) }\end{array}$ \\
\hline 87 bulls & 2021 cows & $0.687 \pm 0.006$ & $0.702 \pm 0.005$ & $0.775 \pm 0.004$ \\
87 bulls and 101 cows & 1920 cows & $0.831 \pm 0.005$ & $0.704 \pm 0.010$ & $0.812 \pm 0.008$ \\
87 bulls and 404 cows & 1617 cows & $0.878 \pm 0.007$ & $0.720 \pm 0.033$ & $0.829 \pm 0.007$ \\
87 bulls and 1011 cows & 1010 cows & $0.901 \pm 0.007$ & $0.709 \pm 0.008$ & $0.842 \pm 0.009$ \\
\hline \hline
\end{tabular}

compared with BTA28, and hence the average computing time for BTA1 was greater than that for BTA28. Another factor affecting computing time is the proportion of masked SNPs in the test population. With $20 \%$ masked SNPs, fastPHASE needed much more computing time than with 95\% masked SNPs. However, an opposite tendency was observed in BEAGLE, in which the time increased with the increase in masked SNP proportion. For findhap, the influence of masked SNP proportion was negligible when it was $20 \%$ to $80 \%$. However, with $95 \%$ masked SNPs, findhap needed slightly more time than did other missing levels.

\section{Scale of reference population}

Reference population size was taken into account to evaluate the efficiency of imputation methods. As shown in Table 5, the imputation accuracy from BEAGLE and findhap was improved with increased reference population size. Particularly for BEAGLE, its accuracy increased from 0.68 to 0.90 when the reference population was increased from 87 bulls to 87 bulls and 1011 cows, whereas a smaller improvement by $6 \%$ was obtained for findhap. Compared with BEAGLE and findhap, performance of fastPHASE was very stable with the lowest accuracy in most situations; almost no differences were observed when the size of the reference population was increased. It is notable that the accuracy from findhap was $8 \%$ and $10 \%$ higher than those from fastPHASE and BEAGLE when the reference population was composed of 87 bulls only. One possible reason is that the 2021 cows in the test population are daughters of 13 bulls in the reference population, and findhap can utilize the pedigree information.

\section{Discussion}

\section{Imputation accuracy}

There are already several reports on the performance of BEAGLE and fastPHASE (Pei et al., 2008; Li et al., 2009; Marchini and Howie, 2010) that show that fastPHASE performs slightly better than BEAGLE in human populations. However, the population structure in cattle is different, and the data reported by Li et al. (2009) involved only 5\% masked SNPs. In our study, we compared mainly BEAGLE, fastPHASE and findhap in scenarios with a high proportion of masked SNPs in order to evaluate their performance when imputing genotypes from low- to high-density genotyping platforms. Our results indicated that BEAGLE performed best and was most robust almost in all situations. fastPHASE was severely affected by the proportion of masked SNPs, especially when the masked SNP proportion was high. The observation that BEAGLE outperformed fastPHASE is consistent with the results in humans seen in the study by Browning and Browning (2007). The comparison of three methods when imputing genotypes from Illumina Bovine $3 \mathrm{~K}(3 \mathrm{k})$ and BovineLD (7k) to BovineSNP50 (50k) further indicated that BEAGLE yielded higher and more stable accuracy. Our study also found that $7 \mathrm{k}$ chip is an ideal low-density genotyping platform for imputing.

Our results are very similar to those of Berry and Kearney (2011), who imputed genotypes from the $3 \mathrm{k}$ panel to the $50 \mathrm{k}$ panel in Holstein-Friesian cattle using Beagle. The concordance between the actual and imputed genotypes in the test group of animals did not vary across chromosomes and was on average $95 \%$. As they used a larger reference population with 4732 animals and 764 animals as the test group, the accuracy they obtained was slightly higher than ours. Similar to BEAGLE, fastPHASE also uses an HMM approach but with several differences that have implications for speed and accuracy. fastPHASE fixes the number of haplotype clusters in the model, whereas BEAGLE is more flexible and dynamically varies the number of clusters at each region. findhap was originally designed for genotype imputation in livestock and takes pedigree information into account. However, it seems that for dairy cattle these could not make it better than BEAGLE. The possible reason for BEAGLE's outperformance is that the HMM is to some extent superior to other algorithms (Li et al., 2009), and the improvement in accuracy from pedigree information is tiny when $L D$ is high enough.

\section{Marker density}

Some investigators observed that imputation accuracy increased with higher marker density (Pei et al., 2008; Druet and Georges, 2010). With denser genotyped markers around missing SNPs, more information could be used to construct haplotypes and deduce the missing SNPs, and therefore better imputation efficiency is expected. As the slope of imputation accuracy decreased markedly with increasing marker density, the benefit of increasing marker density is more obvious for low-density regions (Druet et al., 2010).

The effect of marker density is essentially transformed into that of LD by the fact that denser markers usually cause stronger patterns of local LD, which will help improve 
imputation accuracy (Yu and Schaid, 2007). The result of imputation is population dependent, as different test populations have different LD levels. For example, the LD level $\left(r^{2}\right)$ in Jersey is 0.38 (Weigel et al., 2010), whereas it is about 0.25 in our test population. Using almost the same imputation approach (fastPHASE with 30 haplotype clusters), when masked SNP proportions were $20 \%, 80 \%$, and $95 \%$, the imputation accuracies reported by Weigel et al. (2010) were $0.017,0.034$, and 0.065 higher than those obtained by us, respectively.

Compared with the SNPs distributed in the middle of a chromosome, imputation errors appeared more often in SNPs located at the beginning or end of the chromosome. This is because the marker imputation relies on surrounding markers, whereas the imputation for SNPs at both ends can use information only from one side. Druet et al. (2010) observed $2 \%$ higher error rates at both ends than in the middle of a chromosome in Dutch Holstein, especially for markers in the first or last 10 SNPs of a chromosome. In our study, we also found that the mean imputation error rate for markers at the first or last $5 \%$ SNPs of a chromosome was $0.04 \%$ to $0.4 \%$ higher than that for markers in the middle of the chromosome. Boichard et al. (2012) found that increased concentrations of SNPs at the ends of the chromosomes were helpful to improve imputation accuracy; it was an additional reason for BovineLD imputation to be more accurate than Bovine3K imputation, in addition to the increased overall density of the BovineLD chip compared with Bovine3K.

\section{Computing time}

The vital advantage of findhap is that it saves an enormous amount of computing time. VanRaden et al. (2011) reported that findhap took $2 \mathrm{~h}$ for 33414 Holsteins with 43385 actual markers, and for the same population the time increased only by $2.5 \mathrm{~h}$ even with 500000 simulated markers. Compared with findhap, fastPHASE and BEAGLE run much slower, as they are both localized haplotype clustering-based HMM approaches, and a large amount of time is required for Monte Carlo Markov Chains (MCMC) iterations. Between fastPHASE and BEAGLE, fastPHASE requires substantially more intensive computation, as it takes into account all observed genotypes when imputing each missing genotype, whereas BEAGLE usually focuses on genotypes for a small number of nearby markers when imputing each missing genotype, which makes BEAGLE computationally more efficient (Li et al., 2009). This is further supported by our results. Moreover, the number of haplotype clusters also influences the computing time of fastPHASE severely. Our results showed that when the haplotype clusters were increased from 20 to 30 the computing time was doubled, although the improvement in accuracy was slight. Therefore, the balance between computing time and imputing efficiency for fastPHASE should be considered.

Generally speaking, the magnitude of the computing time in fastPHASE is due to its EM algorithm, which is used to estimate genetic parameters and haplotype frequencies, whereas BEAGLE just adapts empirical frequencies to save computing time. However, with less SNP genotypes, the step of EM to find final likelihoods and parameters accelerated in fastPHASE. Because BEAGLE produces posterior genotype probabilities for imputed genotypes, when the number of ungenotyped SNPs is increased, the amount of calculation, including sampling haplotypes and producing posterior genotype probabilities, is correspondingly increased. As shown in our results, although fastPHASE runs slowest in all situations, the computing time was shortened with the increase in the masked SNP proportion, whereas the computing time for BEAGLE and findhap was increased. One alternative solution to decreasing the computing time for BEAGLE and fastPHASE is parallel processing of the chromosome, as findhap implements a parallel computing algorithm.

\section{Reference population size}

For the situation with $95 \%$ missing SNPs, we changed the reference population size to investigate its influence on imputation accuracy. The size of the test population was correspondingly decreased when the reference population size was increased. The results showed that enlarging the reference population improved the imputation accuracy for BEAGLE but did not affect fastPHASE. Similar results were found by Zhang and Druet (2010); in their study, the imputation accuracy of DAGPHASE (Druet and Georges, 2010) increased when the number of reference individuals was increased, whereas with CHROMIBD (Druet and Farnir, 2011) almost no differences were observed. Druet et al. (2010) observed only small gains in imputation accuracy when the reference population was enlarged with $>1000$ genotyped individuals. In addition to the reference population size, the relatedness among animals in the reference and test population also affects the imputation accuracy; although we did not consider it in this research, it can be done in future studies.

In summary, BEAGLE performed the best and was robust in genotype imputation compared with fastPHASE and findhap. In the scenario of low-masked SNP proportion, fastPHASE, findhap and BEAGLE were comparable, and findhap was more efficient because of its fast computing algorithms. Although fastPHASE and findhap performed worse with the increase in proportion of missing SNPs, BEAGLE continued to maintain higher accuracy in such situations; it is more reliable for imputing genotypes from low- to high-density genotyping platforms.

\section{Acknowledgment}

The project was supported by Ph.D. Programs Foundation of Ministry of Education of China (Grant No. 20110008110001) and the Recommend International Advanced Agricultural Science and Technology Plan of China (Grant No. 2011-G2A), National Dairy Industry System in Beijing Team, Scientific Research Foundation for Returned Scholars, Ministry of Education of China, Program for Changjiang Scholar and Innovation Research Team in University (Grant No. IRT1191). 


\section{References}

Berry DP and Kearney JF 2011. Imputation of genotypes from low- to high-density genotyping platforms and implication for genomic selection. Animal 10, 1-8.

Boichard D, Chung H, Dassonneville R, David X, Eggen A, Fritz S, Gietzen KJ, Hayes BJ, Lawley CT, Sonstegard TS, Van Tassell CP, VanRaden PM, ViaudMartinez KA and Wiggans GR 2012. Design of a bovine low-density SNP array optimized for imputation. PLOS ONE 7, e34130.

Browning SR and Browning BL 2007. Rapid and accurate haplotype phasing and missing-data inference for whole-genome association studies by use of localized haplotype clustering. American Journal of Human Genetics 81, 1084-1097.

Druet T and Farnir FP 2011. Modeling of identity-by-descent processes along a chromosome between haplotypes and their genotyped ancestors. Genetics $188,409-419$

Druet T and Georges M 2010. A Hidden Markov Model combining linkage and linkage disequilibrium information for haplotype reconstruction and quantitative trait locus fine mapping. Genetics 184, 789-798.

Druet T, Schrooten C and de Roos AP 2010. Imputation of genotypes from different single nucleotide polymorphism panels in dairy cattle. Journal of Dairy Science 93, 5443-5454.

Li Y, Willer C, Sanna S and Abecasis G 2009. Genotype imputation. Annual Review of Genomics and Human Genetics 10, 387-406.

Li Y, Willer CJ, Ding J, Scheet P and Abecasis GR 2010. MaCH: using sequence and genotype data to estimate haplotypes and unobserved genotypes. Genetic Epidemiology 34, 816-834.

Marchini J and Howie B 2010. Genotype imputation for genome-wide association studies. Nature Review Genetics 11, 499-511.

Marchini J, Howie B, Myers S, McVean G and Donnelly P 2007. A new multipoint method for genome-wide association studies by imputation of genotypes. Nature Genetics 39, 906-913.
Marshall TC, Slate J, Kruuk LEB and Pemberton JM 1998. Statistical confidence for likelihood-based paternity inference in natural populations. Molecular Ecology 7, 639-655.

Nothnagel M, Ellinghaus D, Schreiber S, Krawczak M and Franke A 2009. A comprehensive evaluation of SNP genotype imputation. Human Genetics 125 , 163-171.

Pei YF, Li J, Zhang L, Papasian CJ and Deng HW 2008. Analyses and comparison of accuracy of different genotype imputation methods. PLOS ONE 3 , e3551.

Qin ZS, Niu T and Liu JS 2002. Partition-ligation-expectation-maximization algorithm for haplotype inference with single-nucleotide polymorphisms. American Journal of Human Genetics 71, 1242-1247.

Scheet $P$ and Stephens M 2006. A fast and flexible statistical model for large-scale population genotype data: applications to inferring missing genotypes and haplotypic phase. American Journal of Human Genetics 78, 629-644.

Shriner D, Adeyemo A, Chen G and Rotimi CN 2010. Practical considerations for imputation of untyped markers in admixed populations. Genetic Epidemiology 34, 258-265.

VanRaden PM, O'Connell JR, Wiggans GR and Weigel KA 2011. Genomic evaluations with many more genotypes. Genetic Selection Evolution 43, 10.

Weigel KA, Van Tassell CP, O'Connell JR, VanRaden PM and Wiggans GR 2010 Prediction of unobserved single nucleotide polymorphism genotypes of Jersey cattle using reference panels and population-based imputation algorithms. Journal of Dairy Science 93, 2229-2238.

Yu Z and Schaid DJ 2007. Methods to impute missing genotypes for population data. Human Genetics 122, 495-504.

Zhang Z and Druet T 2010. Marker imputation with low-density marker panels in Dutch Holstein cattle. Journal of Dairy Science 93, 5487-5494. 